\title{
L’homme fini : un inédit de Gérard Bessette
}

\author{
Guy Monette
}

Collège militaire de Kingston

Ceux qui ont bien connu Bessette se souviennent de l'agacement qu'il manifestait lorsque l'on parlait de "l'éternel Libraire ». Sans doute jugeait-il que l'attention accordée à ce roman, qui est devenu un classique de la littérature québécoise et qu'on a transformé en héraut de la Révolution tranquille, empêchait de voir le reste de son œuvre ${ }^{1}$.

\footnotetext{
${ }^{1}$ Je dédie le présent travail à une personne qui a énormément contribué au rayonnement de l'œuvre de Gérard Bessette et qui persévère dans cette entreprise, mais surtout (et c'est plus important à mon avis) à une personne qui a aimé Bessette et lui a rendu la vie plus agréable, à une personne de qui j'ai eu l'impolitesse de dire à Bessette, et je le prie de me pardonner rétrospectivement, qu'il ne la méritait pas. Il s'agit d'Irène Bakovska Bessette.
} 
Je m'arrêterai donc à un inédit de Bessette intitulé L'homme fini. La trame narrative en est assez simple. Un ancien professeur de littérature y raconte comment il s'est vengé de ses anciens employeurs et de tout un système social dont ils sont les garants :

Voilà sans doute ce qui m'enorgueillissait le plus. Car du ressentiment, de la rancune, inutile de dire si j'en éprouvais. La visite de M. le curé avait fait remonter d'un bloc dans ma conscience une constellation de rancœur. Je me rappelais mes années de collège, mes ruses pour lire des livres tabous, mes remords après les avoir parcourus; tous ces tourments inutiles qui sont épargnés à la jeunesse de la plupart des autres pays et qui retardent si tragiquement notre maturation. (Inédit A, p. 46)

Ce professeur-narrateur décide donc de mettre tout son savoir au service de sa cause : "Sept années durant, j'ai tenté sans succès d'enseigner quelques préceptes à des troupeaux d'ânonnants potaches. Il s'agit de voir si ces préceptes, d'ailleurs inanes, je paux [sic] les appliquer. » (Inédit A, p. 11) Le ton du texte est typique du Bessette première manière, et son intrigue rappelle souvent celle du Libraire. Il contient toutefois un étonnant retour en arrière. Cette irruption du refoulé n'est pas sans rappeler le retour en arrière qui se trouve dans La commensale et au sujet duquel Bessette formulait des réserves qui l'ont d'abord incité à ne pas publier ce roman, ce qu'il fit pourtant en 1975. Je ne résiste pas, en dépit de sa longueur, à citer cet extrait de L'homme fini

En un éclair, je me revis au collège [Sainte-Barbe] (Inédit $A$, p. 67), dans le bureau du préfet des études, un gros père à face rubiconde, qui tenait à la main un livre - La cousine Bette - que j'avais passé à un de mes étudiants qui manifestait une véritable fringale pour les choses littéraires. Et j'entendis la voix de ce même préfet, sèche, dogmatique, qui à la suite d'une harangue à la Savonarole me signifiait mon congé, à moi qui 
enseignais là depuis dix ans! Et je sentis encore le mouvement que je fis pour saisir l'encrier sur le bureau du père et le lui lancer de toutes mes forces. L'encrier le manqua de justesse et alla plaquer une éclaboussure noire sur le mur de crépi blanc. Un souvenir que j'avais refoulé au fond de moi... Me passant la main sur le front, je revins au sentiment de la réalité : le capharnaüm, les livres, le parton [sic] qui, crispé, l'œil haineux, me regardait. (Inédit A, p. 27)

Ce que cet extrait ne dit pas clairement, et que le lecteur aura déjà deviné, c'est que cette éclaboussure noire sur le mur de crépi blanc est, elle-même, devenu un roman : Le libraire.

En effet, L'homme fini [selon la suggestion d'un ami de Bessette, prénommé George, qui est sans doute un ancien collègue de l'université Duquesne ou de l'université de Saskatoon, suggestion basée sur un passage du Libraire (p. 153154)] n'est qu'un des titres qu'aurait pu porter Le libraire, et les extraits cités sont tirés de versions antérieures du roman bien connu. Le subterfuge dont je me suis servi au début de ce texte ne visait qu'à piquer la curiosité et à démontrer, si besoin était, la nécessité d'une édition critique du Libraire. Je n'ai pas ici l'intention d'expliquer la portée des variantes que présentent les diverses versions. Je me contenterai de décrire le matériau sur lequel je travaille, de mentionner certaines difficultés que je rencontre, de formuler quelques commentaires mais, surtout, d'inviter des commentaires et suggestions des lecteurs si jamais ils voulaient me les communiquer.

Sans tenir compte de l'intérêt que peut présenter l'étude des diverses versions d'une œuvre, il importe d'établir une édition " canonique » du texte du Libraire. Depuis sa parution chez Julliard en 1960, le roman a été réédité à plusieurs reprises, et il ne semble pas que son auteur soit intervenu dans 
ces rééditions. Or de nombreuses coquilles se sont glissées dans ces rééditions. Fautes d'orthographe, ponctuations aberrantes, erreurs de noms de personnages, etc., sont fréquentes. Curieusement, elles sont même inconstantes dans les exemplaires d'une même réédition. Attribuables sans doute à ces vilains petits lutins qui hantent les ateliers d'impression, elles déparent le texte de Bessette et parfois le faussent. Mon ami Jacques Allard ne m'en voudra pas de donner l'exemple de la première phrase du roman tiré de l'édition qu'il a préparée pour les Éditions du renouveau pédagogique : «Ma première démarche en arrivant à Saint-Joachin, a été de me chercher une chambre. " (1970, p. 3); on devrait lire: «Ma première démarche en arrivant à Saint-Joachin, c'a été de me trouver une chambre. » (1960, p. 7) La disparition du « c' » rend la première phrase du roman grammaticalement incorrecte et fausse le ton familier qui est de mise pour un journal personnel. Et cette édition est loin d'être la plus fautive; d'ailleurs, il faut dire, à la décharge d'Allard, qu'une autre personne est aussi intervenue à tort dans cette édition. Comme le roman a été maintenant tiré à plus de 200000 exemplaires, on comprendra (si l'on songe que j'ai pu repérer dans une réédition jusqu'à 200 coquilles) que le nombre de leçons erronées est fort élevé.

Les documents sur lesquels je travaille présentent euxmêmes plusieurs difficultés. Le ou les manuscrits du Libraire «ont été perdus lors de déménagements ». Il n'existe que quatre tapuscrits que Bessette a déposés (vendus en fait) à la Bibliothèque nationale du Québec. Selon Bessette, il les a lui-même dactylographiés; d'ailleurs la façon de corriger les fautes de frappe est la même dans les quatre versions. Deux ont 103 pages et deux 99 pages. Une seule est datée, du 17 avril 1958. Les tapuscrits sont des copies carbone. Je soupçonne qu'il 
existe ou a existé une cinquième version du roman, ne serait-ce que les épreuves corrigées de la version finale. On trouve dans ces tapuscrits de nombreuses corrections manuscrites, toutes, sauf peut-être une, de la main de Bessette. Les corrections sur un même tapuscrit sont de différentes encres; elles se retrouvent parfois sur différents tapuscrits ou ont été intégrées aux tapuscrits subséquents; toutefois la chose n'est pas constante. Je crois avoir retrouvé l'ordre chronologique des quatre tapuscrits (Inédits $\mathrm{A}, \mathrm{B}, \mathrm{C}$ et D) quoique certains doutes subsistent quant à cet ordre. D'autre part, les tapuscrits présentent un nombre assez élevé de coquilles et de fautes de frappe. Comment faut-il les traiter dans une édition critique? Et que dire au sujet des corrections tapuscrites qui consistent à dactylographier des « $\mathrm{x}$ » majuscules sur la partie éliminée? Dans ce cas, il est souvent impossible de déchiffrer le texte ainsi corrigé. L'éditeur ou l'auteur a aussi apporté certains changements très mineurs au texte du plus récent tapuscrit; par exemple, la version publiée du roman ajoute un « $F$ » pour indiquer que la température est donnée en degrés Fahrenheit.

Je crois pouvoir expliquer certaines variantes que je qualifie de « francissonnes ». Elles apparaissent presque toutes dans les deux dernières versions. Dans un article intitulé «Publier dans l'exiguïté : le cas du Libraire de Gérard Bessette », j'ai retracé l'histoire de la publication du roman. J'y montre que Gallimard a refusé de publier le roman, entre autres, parce que «[...] des idiotismes canadiens un peu trop nombreux alourdissent inutilement le récit » (2000, p. 447). Dans les deux dernières versions, Bessette élimine beaucoup de ces idiotismes. En voici quelques-uns : «habitants» devient «campagnards », «stand de taxi » : « poste de taxi », « ça me fait pas un pli » : «ça me fait ni chaud ni froid », «que je baraude » : « que je traîne ma carcasse », 
ou encore « coupe-vent de cuir » qui devient d'abord «blouson » et finalement «vareuse ». D'ailleurs une de ces francissonneries m'avait toujours agacé bien avant que je prenne connaissance des tapuscrits, et j'en avais parlé à Bessette. On se souvient sans doute que, un soir, Rose se rend à « une quelconque partie de loto à la salle paroissiale » (1960, p.129). Cette partie de loto me paraissait peu appropriée dans un contexte nord-américain. La leçon que donne le premier tapuscrit est évidemment « un quelconque bingo à la salle paroissiale » (Inédit A, p. 72). La correction manuscrite « une quelconque partie de loto » apparaît dans les tapuscrits subséquents. Quand je demandai à Bessette pourquoi il ne remplaçait pas le mot «loto» par le mot «bingo», une moue difficile à déchiffrer fut sa seule réponse. J'ai donc écrit dans «Publier dans l'exiguïté : le cas du Libraire de Gérard Bessette » :

On [il faut maintenant lire Bessette] a sacrifié le jeu de bingo inconnu en France, au jeu de loto, qui lui est inconnu au Canada. Cependant on peut s'étonner que ni l'auteur ni l'éditeur canadien n'aient rétabli le mot «bingo » dans les réimpressions canadiennes du roman. (2000, p. 451).

Si édition critique il y a, faudrait-il le faire? J'inclinerais en ce sens. Toutefois, qu'en est-il des autres francissonneries, et où doit-on s'arrêter? Pour le moment, la version canonique du roman est pour moi l'édition Julliard. Ai-je tort? Je me permets à ce sujet une remarque sur Les anthropoïdes. À la toute fin de ce roman, le nom « Kébékouâ » désigne un «fleuve géant » (1977, p. 295) déjà évocateur puisque cette expression désigne le Saint-Laurent dans l'hymne national du Canada. Lors d'une discussion, j'affirmai à Bessette que le mot «Kébécois» me paraissait une concession facile à une mode "nationaleuse » et inciterait le lecteur à chercher des clefs dans le roman. Bessette 
me répondit : «Si le roman est réédité, je le supprimerai. » L'avenir devra-t-il tenir compte de cette information?

Afin de me faire pardonner mon léger coup de griffe, élimée certes, à Jacques Allard, ce critique apprendra avec plaisir que les tapuscrits confirment ses sagaces remarques quant au fait que Jodoin évite soigneusement de citer directement ses propres paroles. Les quelques endroits où il le faisait dans les tapuscrits ont été soigneusement élagués : « Je suis pour la liberté, dis-je. » (Inédit A, p. 90) devient par la suite : « Je lui déclarai que j’étais pour la liberté. » (Inédit B, p. 93) Grâce à cette technique, Jodoin se protège « des cinglures du monde extérieur par cette espèce de matelas psychologique que je connais si bien » (Inédit B, p. 72); mais, surtout, il peut exercer son ironie en toute impunité. Quant à la longue et unique tirade en style direct de Jodoin (1960, p. 52; Inédit A, p. 27), il importe de souligner qu'elle suit immédiatement le passage du retour en arrière déjà cité. Sans tenir compte du fait que l'élimination de ce retour en arrière permet de conserver à Jodoin son caractère lymphatique et mystérieux, ne toucherait-on pas ici à une des émergences hâtives, comme c'était le cas pour le retour en arrière de La commensale, du Bessette deuxième manière, beaucoup plus intéressé aux mouvements de la psyché de ses personnages qu'au réalisme traditionnel?

Mais je m'éloigne par trop de mon sujet et en reviens aux simples variantes et aux malices vengeresses du professeur congédié. On sait que Jodoin n'a pas le curé de Saint- Joachin à la bonne. Lorsque les pères du collège Saint-Rock (parfois désigné par un Sainte-Barbe trop limpide) jouent un sale tour à ce saint homme, Jodoin écrit d'abord qu'il a fallu « un mois » à M. Galarneau pour saisir ce qui se passait; une correction tapuscrite indique par la suite qu'il lui a fallu en fait « six mois » 
(Inédit A, p. 85). Pas futé ce curé! Toutefois Jodoin atténue son agressivité lors de la visite du curé à la librairie : «J'espérais lui passer un rossignol, en le lui faisant payer - cela va sans dire - deux fois le prix marqué. » (Inédit A, p. 45) deviendra simplement : «J'espérais lui refiler un rossignol. » (1960, p. 83) En revanche, ses sentiments plus affectueux envers d'autres personnages sont atténués. Ainsi à propos du père Manseau, il éliminera un « C'est peu mais c'est quelque chose. » (Inédit B, p. 30) après « $\mathrm{Au}$ moment de partir, en se levant, il grogne de nouveau, et moi, je lui dis au revoir » $(1960$, p. 56).

Je ne m'attarde pas ici à ce qu'on pourrait appeler des corrections stylistiques, assez peu nombreuses d'ailleurs, et me contente d'en donner un exemple : «À la mort du père Rivard (tel était le nom du prédicateur), il s'en était, toujours comme par hasard, trouver un autre pour le remplacer et continuer la tradition. » (Inédit B, p. 86) devient : « À la mort du père Rivard (tel était son nom), on avait trouvé, toujours comme par hasard, un autre prédicateur éloquent et spirituel pour le remplacer et continuer la tradition » (1960, p. 151-152). Ce qui m'intéresse surtout dans cet exemple, c'est le déplacement du mot "prédicateur »; l'ajout de deux adjectifs et la polysémie de «spirituel» ajoutent toutefois beaucoup au texte final. Le passage de " pouvant contenir » (Inédit B, p. 85) à « capable de contenir » (1960, p. 150) serait sans doute un meilleur exemple de variante plus nettement stylistique.

Comment devrait se présenter l'édition critique du roman? Faudra-t-il indiquer la moindre variante, la moindre correction? Les regrouper en catégories? Comment agencer les notes? Quel type de bibliographie devrait l'accompagner? Faut- 
il tenir compte des coquilles des diverses éditions? Voilà autant de questions que je me pose.

Comme on le constate, il reste encore beaucoup à faire, et pas seulement en ce qui concerne Le libraire. Par exemple, on trouvera dans le fonds Gérard Bessette de la BAnQ le manuscrit de plus de mille pages des Anthropoïdes. Une analyse de ce manuscrit serait un bon sujet de thèse. Le reste des manuscrits de l'auteur, que Mme Bessette a déposé dans le fonds Gérard Bessette en 2006, attend encore d'être inventorié. Il est toutefois dommage que, selon la volonté de l'auteur, son journal et sa correspondance ne deviennent accessibles que dans 49 ans.

Lorsque j'ai entrepris ce projet d'édition critique du Libraire, je croyais que la tâche serait facile et ne demanderait que quelques semaines. Comme Jodoin, j'aime travailler « pourvu qu'il n'y ait rien à faire » (1960, p. 23). Avec sa gentille malice, j'imagine que Bessette a prévu les affres que son œuvre susciterait chez nous, pauvres critiques, lorsqu'il a écrit Les dires d'Omer Marin. Mais, finalement, peu importe les méthodes critiques utilisées, ce qui compte en littérature, c'est de dialoguer avec une œuvre et un écrivain, parfois en suant, parfois en sacrant, parfois en riant, somme toute, en continuant à vivre et à les faire vivre.

\section{Bibliographie}

BESSETTE, Gérard (1960), Le libraire, Paris, Julliard.

- (1970), Le Libraire, Montréal, Éditions du Renouveau Pédagogique, coll. « Lecture Québec ». 
- (1975), La commensale, Montréal, AS/Quinze.

- (1977), Les anthropoïdes, Montréal, Les Éditions La Presse.

MonetTE, Guy (2000), "Publier dans l'exiguïté: Le cas du Libraire de Gérard Bessette» dans Robert Viau (dir.), $L a$ création littéraire dans le contexte de l'exiguité, Beauport, Publications MNH, coll. «Écrits de la francité », p. 439-453.

\title{
Résumé
}

Cet article plaide en faveur d'une édition critique du Libraire. Cet ouvrage a en effet été précédé par divers brouillons et romans inachevés disponibles dans le fonds Gérard Bessette déposé à la BAnQ notamment $L$ 'homme fini. Certains passages de cet inédit annoncent clairement ce qui deviendra le plus célèbre roman de Gérard Bessette. En outre, comme Le libraire a été publié originalement à Paris, diverses corrections de langue et de style ont été faites pour le public français. Une édition critique permettrait de les signaler pour le lecteur contemporain.

\begin{abstract}
This article illustrates the need for a critical edition of $L e$ libraire, a novel that was preceded by various drafts and unfinished novels that are now available in the Fonds Gérard Bessette at the BAnQ, most notably L'homme fini. Various passages of this unpublished novel clearly announce what will become the most famous novel by Gérard Bessette. Moreover, since Le libraire was originally published in Paris, the vocabulary and style were edited for French readers. A critical edition would allow the contemporary reader to become aware of these often significant variations.
\end{abstract}

